\title{
APLICAÇÃO DO MÉTODO ANALÍTICO À INDICATRIZ UNIAXIAL *
}

\author{
IBRAHIM OCTAVIO ABRAHÃO** \\ ARARY MARCONI **
}

\begin{abstract}
RESUMO
O método analítico é estudado, em seus aspectos teóricos, visando a sua aplicação em minerais uniaxiais. A marcha de cálculo reduz-se a uma equação de segundo grau, em que ambas as raízes representam posições possíveis do eixo ótico. São fornecidas três maneiras diferentes para se fazer a opção entre as duas raízes. Estuda-se também a determinação da birrefringência máxima, cuja expressão, para os uniaxiais, assume formas muito simples. Conclui-se, finalmente, que o sinal ótico do mineral é o mesmo sinal da birrefringência da secção tomada na posição inicial de extinção $(\varphi=\theta=\Psi=0)$.
\end{abstract}

\section{INTRODUÇÃO}

O método analítico vem recebendo atenção dos autores, que constataram a viabilidade de sua aplicação a secções delgadas de minerais biaxiais em uma série de trabalhos. Os autores não têm conhecimento de nenhum trabalho sobre sua aplicação a minerais uniaxiais desde sua criação. $O$ presente trabalho trata dos aspectos teóricos da aplicação do método a minerais uniaxiais em secção delgada, à luz dos resultados encorajadores sobre o método no seu emprego a biaxiais.

O método analítico, cuja complicação de cálculo parece aos autores ter sido a principal causa que impediu sua aplicação até o advento dos modernos recursos da computação elettônica, assume feições particularmente simples no caso de minerais uniaxiais. Dessa forma, a expectativa dos autores é de que sua aplicação conduza a vantagens de ordem operacional na rotina de levantamento da posição do eixo ótico e na determinação da grandeza da indicatriz, especialmente no que se refere à máxima birrefringência da in dicatriz uniaxial.

\section{REVISÃO DA LITERATURA}

Apenas CHOMARD (1934) trabalha com uniaxiais na aplicação do método analítico. Mostra que, se para $\varphi=0^{\circ}$ (ou $\varphi=90^{\circ}$ ) tem-se $\Psi=0^{\circ}$ para qualquer $\theta$, mas não para $\varphi=90^{\circ}$ (ou $\varphi=0^{\circ}$ ), o mineral deve ser encarado aprioristicamente como uniaxial. Estabelece as simplificações que resultam no cálculo envolvido e mostra que a marcha de cálculo acaba por reduzir-se a uma equação do segundo grau, $\mathrm{q}^{2}+\mathrm{qktq}-\mathrm{k}^{2}=0$, em que $\mathrm{k}=\operatorname{sen} \theta$ e $\mathrm{t}=\operatorname{cotg} 2 \Psi$, de cujas raízes apenas uma deve corresponder à solução

* Entregue para publicação em 13/10/1975.

* Departamento de Solos e Geologia, ESALQ - USP. 
$\mathrm{q}=\operatorname{tg} \delta$, em que $\delta$ é o ângulo que o eixo ótico faz com a normal à lâmina. Sugere, então, que se façam pelo menos duas experiências de extinção $(\theta, \Psi)$, conduzindo às equações:

$$
\begin{aligned}
& q^{2}+2 k_{1} t_{1} q-k_{1}^{2}=0 \\
& q^{2}+2 k_{2} t_{2} q-k_{2}^{2}=0, \text { cuja solução comum é } \\
& t=q=\frac{k_{2}^{2}-k_{1}^{2}}{2\left(k_{2} t_{2}-k_{1} t_{1}\right)}
\end{aligned}
$$

Operacionalmente, sugere que se façam $n$ operações de extinção, a cada uma das quais corresponde uma equação de segundo grau, com duas raízes $q_{1}$ e $q_{2}$. Uma deve ter o mesmo valor em todas as equações e a outra valor variável. A média das $\mathrm{n}$ raízes de valor aproximadamente igual seria a solução procurada $q=\operatorname{tg} \delta$. Executa, então, 9 experiências de extinção em um grânulo de quartzo $(\theta=5,10,15,20,25,30,35,40$, $45^{\circ}$ ), mostrando, efetivamente que uma das raizes tem valor constante e a outra variável. Além disso, determina o valor de $\mu \lambda$ e calcula $\lambda$ pela medição de $\mu$ e o índice principal $\mathrm{Ng}$ por $\mathrm{Ng}=\mu+\mu(1+\cos 2 \mathrm{~V})$.

ABRAHÃO (1968) retoma o método e mostra sua validade na determinação da posição da indicatriz de plagioclásios, valendo-se de um programa para computador. A partir de então alguns trabalhos têm sido publicados sobre a aplicação do método a biaxiais. Alguns, de caráter operacional e estatístico, visam ao emprego do método na rotina de trabalho: ABRAHÃO (1971, 1974a, 1974b); ABRAHÃO e GODOY (1971); outros, de caráter mais teórico, mostram as possibilidades de cálculo inteiramente analítico da indicatriz, em posição e grandeza: ABRAHÃO e MARCONI (1974a, 1975a, 1975b).

\section{LEVANTAMENTO ANALITICO DA INDICATRIZ}

A situação de uma seç̧ão uniaxial, no sistema de eixos usado na simplificação do método (posição inicial de extinção, ABRAHÃO, 1968) é a ilustrada nas figs. 1 e 2 , que se traduz nas expressões:

$$
\begin{array}{lll}
\mathrm{j}=\operatorname{sen} \delta & \mathrm{p}=\mathrm{p}^{\prime}=0 & \mathrm{pq}^{\prime}+\mathrm{p}^{\prime} \mathrm{q}=0 \\
\mathrm{~m}=0 & \mathrm{p}+\mathrm{p}^{\prime}=0 & \\
\mathrm{n}=\cos \delta & \mathrm{q}=\mathrm{q}^{\prime} &
\end{array}
$$




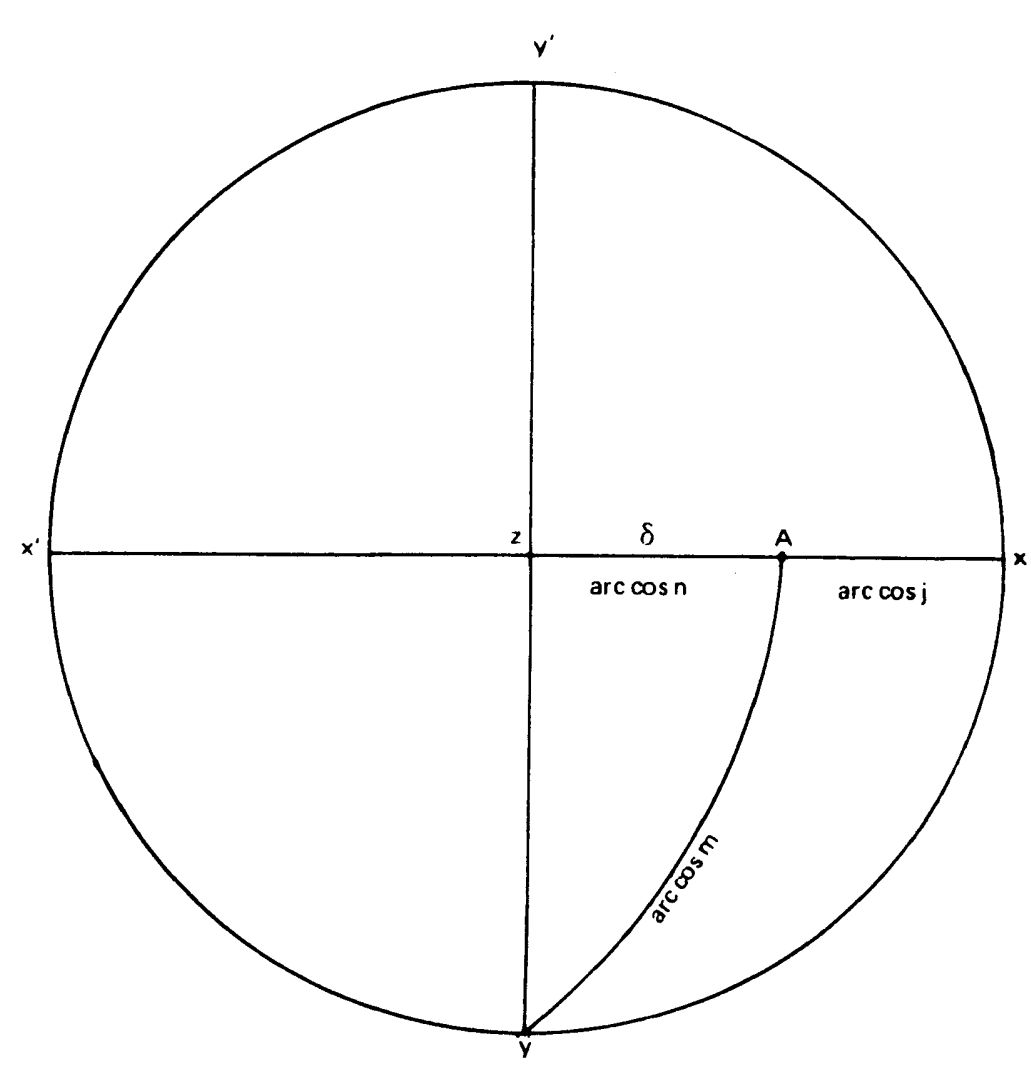

Fig. 1

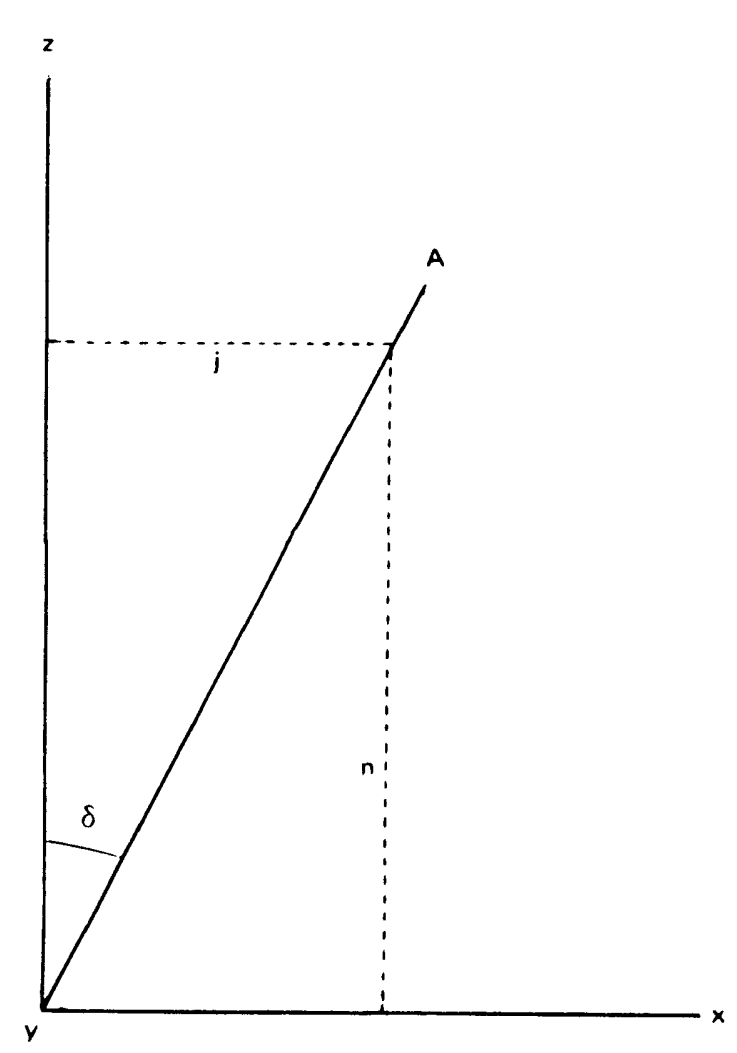

Fig. 2

A expressão geral a que deve satisfazer toda operação de extinção $(\varphi, \theta, \Psi)$ dos biaxiais (ABRAHÃO, 1968) reduz-se, então, a:

$$
\left(v^{2}-h^{2} u^{2}-2 u v h t\right) q^{2}+2 k(u h+v t) q-\left(u^{2}-v^{2}\right)\left(1-h^{2}\right)=0,
$$

$$
\begin{aligned}
& \text { em que } \mathrm{u}=\cos \varphi \quad \mathrm{h}=\cos \theta \\
& \mathrm{v}=\operatorname{sen} \varphi \quad \mathrm{k}=\operatorname{sen} \theta \text {, donde resulta: } \\
& \mathrm{q}^{2}+2 \mathrm{ktq}-\mathrm{k}^{2}=0
\end{aligned}
$$

\section{Cálculo da posição do eixo ótico}

Sejam $q_{1}$ e $q_{2}$ as raízes de (2), das quais, segundo CHOMARD (1934), apenas uma corresponde à realidade física, isto é, exprime a tangente do ângulo do eixo ótico com a vertical. Não é essa, porém, a interpretação correta. Ambas as raizes têm significado físico e indicam que há 2 posições de eixo ótico que produzem o mesmo ângulo de extinção $\Psi$, após a rotação $\theta$. Com efeito, como o eixo ótico está necessariamente sobre Xx' (fig. 3), se uma rotação $\theta$ leva $A_{1}$ sobre $O_{1}\left(A_{1}^{\prime}\right)$, produzindo o ângulo de extinção $\Psi$, algum eixo ótico $\mathrm{A}_{2}$, pela mesma rotação $\theta$ cairia sobre $\mathrm{OM}_{2}\left(\mathrm{~A}_{2}^{\prime}\right)$ e produziria o mesmo ângulo de extinção $\Psi$. Observa-se que para $\Psi<45^{\circ}, \delta_{1}>\delta_{2}$ e, para $\Psi<45^{\circ}$, $\delta_{1}<\delta_{2}$. As soluções da equação (2) são precisamente $q_{1}=\operatorname{tg} \delta_{1}$ e $q_{2}=\operatorname{tg} \delta_{2}$. 


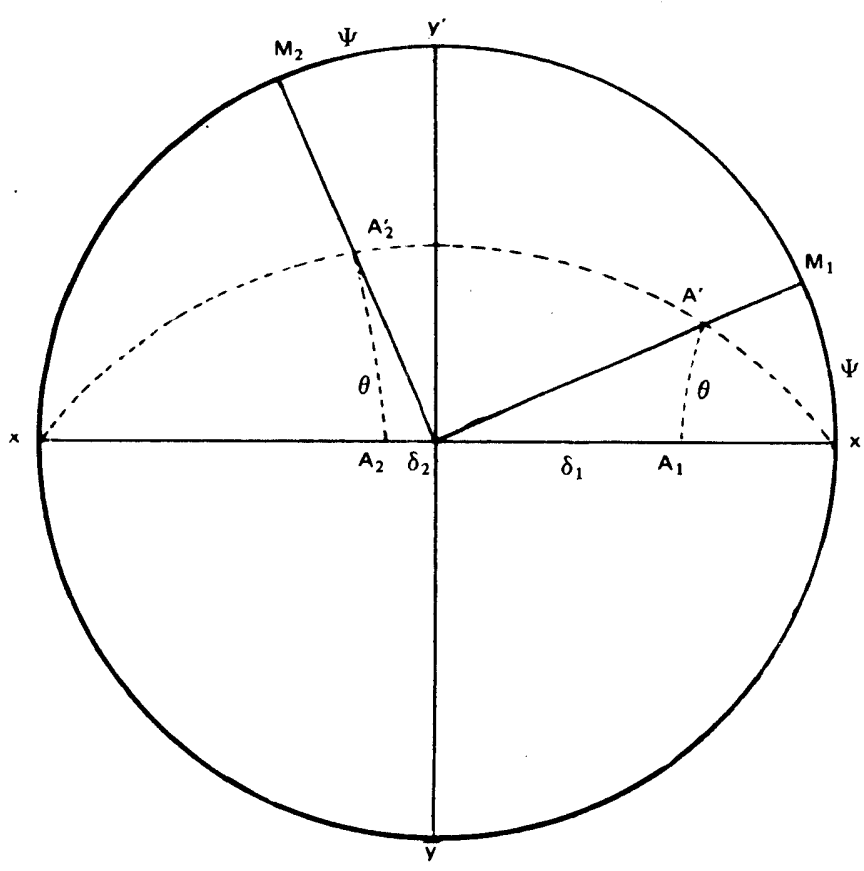

Fig. 3

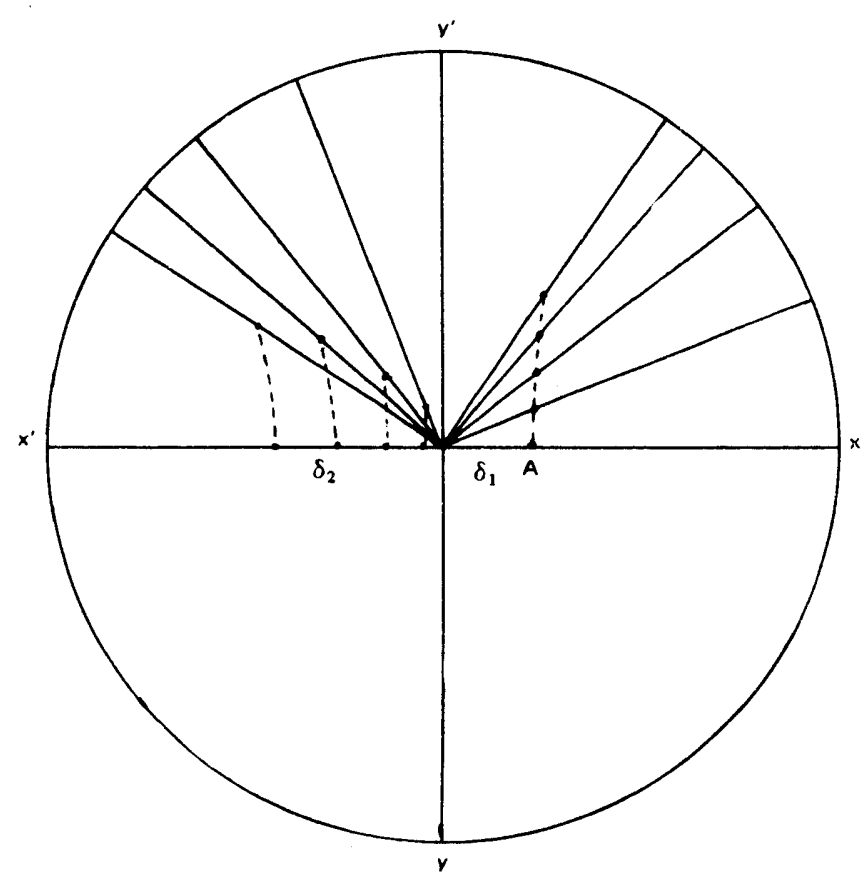

Fig. 4

Efetuemos, à guisa de exemplo, as seguintes operações de extinção na secção uniaxial da figura 4.

\begin{tabular}{rccccr}
\hline$\theta$ & $\Psi$ & $\mathrm{q}_{1}$ & $\mathrm{q}_{2}$ & 1 & \multicolumn{1}{c}{2} \\
\hline 10 & 21,0 & $-0,452$ & 0,066 & $-24,335$ & 3,818 \\
20 & 37,5 & $-0,445$ & 0,262 & $-24,018$ & 14,710 \\
30 & 48,0 & $-0,450$ & 0,555 & $-24,232$ & 29,048 \\
40 & 55,0 & $-0,450$ & 0,918 & $-24,226$ & 42,556 \\
\hline
\end{tabular}

Observa-se, assim, que, efetivamente, uma das raízes é constante e a outra variável, com sinais opostos. Conclui-se, pois, que a equação (2) fornece as duas soluções possíveis.

É necessário, finalmente, decidir se, no caso em observação, tem-se $\delta_{1}$ ou $\delta_{2}$. Isto pode ser feito, no microscópio, com uma rotação-teste em torno do eixo y, procurando levar a 0 o eixo ótico mais próximo. A questão se resolve, também, no próprio contexto do método. Com efeito, usando-se pelo menos duas equações (2), com o uso, pois de 2 valores para $\theta$, se $\Psi$ aumentar com o aumento de $\theta$, a solução é, necessariamente, $\delta_{1}$, se diminuir, é $\delta_{2}$. Também: se as duas raízes de valor constante são negativas, a solução é $\delta_{1} ;$ se são positivas, será $\delta_{2}$. 


\section{Cálculo da birrefringência máxima}

Nos biaxiais, a equação geral a que devem satisfazer todas as operações de extinção e birrefringência $(\varphi, \theta . \Psi, \mathrm{r})$ é (ABRAHÃO, 1968):

$$
2 \lambda(\alpha-\beta)+6 \lambda^{2}\left(\alpha^{2}-\beta^{2}\right)+20 \lambda^{3}\left(\alpha^{3}-\beta^{3}\right)+\ldots=\mathrm{r} / \mu
$$

em que r é a birrefringência da secção, $\mu=\mathrm{Nm}$ é o índice médio do mineral e

$$
\begin{aligned}
& \alpha=a^{2} j j j^{\prime}+a^{\prime 2} m m^{\prime}+a^{\prime 2} n n^{\prime}+a^{\prime} a^{\prime \prime}\left(m n^{\prime}+m^{\prime} n\right)+a a^{\prime \prime}\left(j n '+j^{\prime} n\right) \\
& \beta=b^{2} j j \text { ' }+b^{\prime 2} m m^{\prime}+b^{\prime \prime 2} n n^{\prime}+b^{\prime} b^{\prime \prime}\left(m n^{\prime}+m^{\prime} n\right)+b b^{\prime \prime}\left(j n^{\prime}+j^{\prime} n\right)
\end{aligned}
$$

Para os uniaxiais, essas expressð̃es reduzem-se, na posição inicial de extinção $\varphi=e=$ $\Psi=0, \mathrm{a} \alpha=\mathrm{j}^{2}=\operatorname{sen}^{2} \delta$ e $\beta=0$.

A expressão $\mu \lambda=\frac{r}{2\left(\mathrm{jj}^{\prime}-\mathrm{mm}\right)}$, válida para a mesma posição e que permite o cálculo de $B=2 \mu \lambda$, birrefringência máxima do mineral, reduz-se, pois, $a \lambda=r / 2 j^{2}$ (4).

Para qualquer outra experiência $(\varphi, \theta, \Psi, \mathrm{r}), \theta \neq 0, \Psi \neq 0$ e $\varphi=0$, levando em " conta que, em (3):

$$
\begin{array}{ll}
a=\cos \Psi & b=\operatorname{sen} \Psi \\
a^{\prime}=-\operatorname{sen} \Psi \cdot \cos \theta & b^{\prime}=\cos \Psi \cdot \cos \theta \\
a^{\prime \prime}=\operatorname{sen} \Psi \cdot \operatorname{sen} \theta & b^{\prime \prime}=-\cos \Psi \cdot \operatorname{sen} \theta \\
\alpha=a^{2} j^{2}+a, " 2 n^{2}+2 a a " j n=(a j+a " n)^{2} \\
\beta=b^{2} j^{2}+b^{\prime \prime 2} n^{2}+2 b b " j n=(b j+b " n)^{2}
\end{array}
$$

A expressão geral da birrefringência máxima $B=2 \mu \lambda=\frac{r}{2(\alpha-\beta)}$ para os biaxiais (ABRAHÃO, 1968) assume, então, nos uniaxiais a forma:

$$
2 \mu \lambda=\frac{r}{(a j+a " n)^{2}-(b j+b " n)^{2}}
$$

\section{Determinação do sinal ótico}

Demonstra-se que, nos biaxiais, o sinal ótico do mineral é o mesmo de $\lambda$ e, dado que $\mu$ é sempre positivo, é também o de $\mu \lambda$ (ABRAHÃO, 1968). Para os uniaxiais, 
observa-se que, em vista de (4), o mineral tem o sinal de r, o que é esperado. Com efeito, se a indicatriz está na posição da figura 1 e é positiva (ou negativa) sobre Oy está o semisixo $N_{1}=N p$ (ou $N_{1}=N g$ ), enquanto sobre Ox estará um semi-eixo $N_{2}=N^{\prime} g$ (ou $\mathrm{N}_{2}=\mathrm{N}^{\prime} \mathrm{p}$ ) tal que N'g $>\mathrm{Np}$ (ou N'p $<\mathrm{Ng}$ ). Portanto, como convencionalmente a birrefringência tem o sinal de $N_{1}-N_{2}$ (ABRAHÃO, 1968), o sinal ótico é o de r. Dessa forma, a determinação de $\mu \lambda$ atende a uma dupla finalidade: torna conhecida a birrefringência máxima do mineral e seu sinal ótico.

\section{CONCLUSÕES}

O emprego do método analítico a secções de minerais uniaxiais tem a vantagem de não exigir, como nos biaxiais, programa para computador, resumindo-se à solução de equações de segundo grau.

Tanto para a posição de extinção inicial, como para qualquer outra operação de extinção e birrefringência, a expressão de birrefringência máxima assume formas muito simples. Ademais, a determinação de $\mathrm{B}=2 \mu \lambda$ fornece também o sinal ótico do mineral.

A determinação da orientação da indicatriz uniaxial é muito simples. Há sempre duas posições possiveis e a opção se faz sempre possivel na própria platina (rotação-teste ao redor de y) ou na variação da grandeza de $\Psi$ com aumento de $\theta$ ou ainda no sinal algébrico da raiz constante.

\section{SUMMARY}

\section{ON THE ANALYTICAL STUDY OF THE UNIAXIAL INDICATRIX}

This paper deals with theoretical considerations on the application of analytical method to uniaxial crystals. All the complicated calculation involved with biaxial crystals is reduced to a second degree equation. Each of its roots represents a possible position of the optical axis. Analytical determination is made, too, for the selection of one of the roots. The determination of total birefringence is studied. Its expression takes very simple analytical forms. It was concluded, finally, that the optical sign of the mineral is the same as the sign of its partial birefringence at the position $\varphi=\theta=\Psi=0$.

\section{LITERATURA CITADA}

ABRAHÃO, I.O., 1968. Contribuição ac Estudo do Método Analítico de Chomard. Tese de LivreDocência, ESALQ, USP, Piracicaba, SP, 132 p.

ABRAHÃO, I.O., 1971. Determinação do Ângulo 2V de Plagioclásios pelo Método Analítico com Valores de $\varphi$ Múltiplos de 90\%. Anais da ESALQ, USP, 28:69-81.

ABRAHÃO, I.O., 1974a. Determinação de 2V em Plar oclásios pelo Método Analítico com Valores de $\varphi$ Múltiplos de $45^{\circ}$. Anais da ESALQ, USP, 31 9-33. 
ABRAHÃO, I.O., 1974b. Sobre a Determinação do Āngulo de Extinção para Aplicação do Método Analítico. Anais da ESALQ, USP, 31:7-18.

ABRAHÃO, I.O. e GODOY, C.R.M., 1971. Solução para Computador do Método Analítico de Chomard. Anais da ESALQ, USP. 28:227-234.

ABRAHÃO, I.O. e MARCONI, A., 1974. Elipsóides Concíclicos. Anais da ESALQ, USP, 31: 225-231.

ABRAHÃO, I.O. e MARCONI, A., 1975a. Determinação Analítica da Indicatriz Ótica de Minerais. Anais da ESALQ, USP. 32.

ABRAHÃO, I.O. e MARCONI, A., 1975b. Sobre a Determinação Analítica da Indicatriz Ótica de Anidrita. Anais da ESALQ, USP. 32.

CHOMARD, L. 1934. Théorie et Pratique de la Méthode Fédorow. Procedé Classique et Méthode Analy tique Générale. Dunod, Paris. Annales des Mines, V:153-218. 
$$
\text { . }
$$ 